\title{
Response to the letter by Matsushita et al. regarding our manuscript "Resection Depth and Layer of Cold Snare Polypectomy versus Endoscopic Mucosal Resection"
}

\author{
Tomoaki Suga ${ }^{1}$ (D) Akihiro Ito $^{2} \cdot$ Hiroyoshi Ota $^{3} \cdot$ Nobuyuki Tateiwa $^{4} \cdot$ \\ Akihiro Matsumoto ${ }^{2} \cdot$ Eiji Tanaka $^{2}$
}

Received: 13 February 2019/Accepted: 26 February 2019/Published online: 9 March 2019

(C) Japanese Society of Gastroenterology 2019

We thank Dr. Matsushita et al. for their comments on our article "Resection Depth and Layer of Cold Snare Polypectomy versus Endoscopic Mucosal Resection". However, it appears that their arguments are based on the perspective of mucosal lesions. In mucosal lesions without submucosal invasion, we agree that the submucosal thickness of the resected specimen is not an important matter because it does not affect treatment outcome. In the clinical setting, however, we have encountered diminutive polyps that were apparently mucosal lesions as well as small polyps of 6-9 $\mathrm{mm}$ in diameter. In another study, small polyps contained $4 \%$ of category 4 or 5 polyps; of those, $6 \%$ were deep invasive cancers with lymph node metastasis requiring further surgical treatment [1]. Moreover, some of the malignant small polyps were nonpolypoid and not easily recognized as invasive before endoscopic resection.

Resected specimens require histopathological evaluation, especially at the vertical margin. For polyps, specimens from CSP contained significantly more unevaluable samples than those resected by EMR in our report. Only precise histopathological assessment at vertical margins

Tomoaki Suga

sugatomo@shinshu-u.ac.jp

1 Endoscopic Examination Center, Shinshu University Hospital, 3-1-1 Asahi, Matsumoto 390-8621, Japan

2 Division of Gastroenterology and Hepatology, Department of Medicine, Shinshu University School of Medicine, Matsumoto, Japan

3 Department of Biomedical Sciences, School of Health Sciences, Shinshu University School of Medicine, Matsumoto, Japan

4 Tateiwa Clinic, Nagano, Japan can identify patients with invasive tumors for proper additional treatment. Simple endoscopic removal of numerous colorectal polyps may indeed reduce the incidence and mortality of CRC, but possibly at the expense of inadequately resected submucosal invasive cancers due to insufficient sampling.

It appears true that EMR specimens tend to have a thicker submucosa as a result of saline injection. However, we maintain that the EMR technique has the ability to resect deeper, and more importantly evaluable, layers than does CSP. This may improve the evaluation of vertical margins in cases of invasive cancer appearing as benign polyps.

\section{Reference}

1. Sakamoto T, Matsuda T, Nakajima T, Saito Y. Clinicopathological features of colorectal polyps: evaluation of the 'predict, resect and discard' strategies. Colorectal Dis. 2013;15:e295-e300.

Publisher's Note Springer Nature remains neutral with regard to jurisdictional claims in published maps and institutional affiliations. 